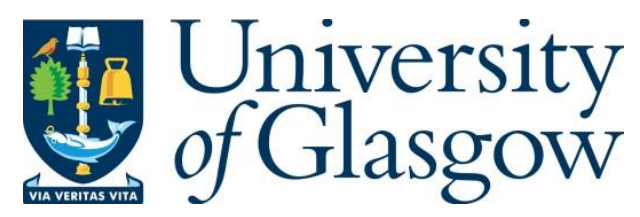

Fatholah nejad, H., Dideban, D., Ketabi, A., Vali, M., Bayani, A. H. and Heidari, H. (2018) Tuning the analog and digital performance of Germanene nanoribbon field effect transistors with engineering the width and geometry of source, channel and drain region in the ballistic regime. Materials Science in Semiconductor Processing, 80, pp. 18-23.

There may be differences between this version and the published version. You are advised to consult the publisher's version if you wish to cite from it.

http://eprints.gla.ac.uk/156709/

Deposited on: 19 February 2018

Enlighten - Research publications by members of the University of Glasgow http://eprints.gla.ac.uk 


\title{
Tuning the analog and digital performance of Germanene nanoribbon field effect transistors with engineering the width and geometry of source, channel and drain region in the ballistic regime
}

\author{
Hossein Fatholah nejad ${ }^{1}$, Daryoosh Dideban ${ }^{2,1, *}$, Abbas Ketabi $^{2}$, Mehran Vali $^{1}$, Amir Hossein \\ Bayani $^{1}$, Hadi Heidari ${ }^{3}$ \\ ${ }^{1}$ Institute of Nanoscience and Nanotechnology, University of Kashan, Kashan, Iran \\ ${ }^{2}$ Department of Electrical and Computer Engineering, University of Kashan, Kashan, Iran \\ ${ }^{3}$ Electronics and Nanoscale Engineering Research Division, School of Engineering, University of \\ Glasgow, Glasgow, UK
}

\begin{abstract}
In this paper, with taking advantage of electrical properties of a germanene nanoribbon, we propose a germanene nanoribbon field effect transistor (GeNRFET). Here by tuning the width and geometry of the germanene nanoribbon in the source, drain and channel regions, we investigate theoretically the transistor characteristics, analog and digital performances of these several different GeNRFETs at room temperature. Our simulations are obtained using density functional theory (DFT) combined with non-equilibrium Green's function (NEGF) method. The simulation results show that for digital applications, by tuning the width of the germanene nanoribbon a GeNR-FET with a finite band gap in the channel region and small band gap in the source and drain regions shows a better $\mathrm{I}_{\text {on }} / \mathrm{I}_{\text {off }}$ ratio in transfer characteristics. However, for the analog applications, if the band gap of the channel region has small value and the band gap of the source and drain regions have a finite value, the output characteristic shows a higher peak to valley (PVR) ratio which is an important figure of merit in analog applications. Also from the output characteristics, we find that the T-shape channel shows more desirable (PVR) compared with other devices and it reaches to 17.28 in this case.
\end{abstract}

\section{Introduction}

Since the experimental fabrication of two-dimensional honeycomb structures of carbon atoms named as Graphene [1-3] and due to its promising electronic and mechanical properties, there has been a vast extent of experimental and theoretical 
research on such 2D materials [4-6]. Among them, there are atomically thin structures of Si and Ge which are called Silicene and Germanene, respectively [710]. As Graphene, These are also honeycomb structures with a Dirac-point electronic band structure. Although planar Germanene is reported to be unstable and metallic but the buckled structure is stable and behaves as a semi-metal [11].

In terms of transistor performance, a single layer of graphene or germanene has zero bandgap which does not allow proper cut-off in the graphene and germanene FETs. We can overcome this issue by confining such materials in one dimension and making nanoribbons or nanotubes. Graphene nanoribbons show considerable electronic properties such as the dependence of the electronic bandgap on the width of the ribbon [12-13]. As the width of the ribbon increases in Graphene, the electrical bandgap decreases [14]. A similar width dependence behavior has been also investigated in Silicene and Germanene nanoribbons [15]. Electrical band gap existence [16-17] and other properties such as high carrier mobility [18-19] and high current density [20] of Graphene and Germanene makes them as excellent candidates in transistor [21] and sensor applications [17]. By changing the width of the nanoribbon and building a field effect transistor whose bandgap in the channel is different from the source and drain regions, it will be possible to obtain a nonlinear effect such as negative differential resistance (NDR). This phenomenon is interesting for designing high-speed analogue devices [22]. Although investigations have been carried out on graphene nanoribbonsFETs [23-24], but no study is yet performed to utilize germanene nanoribbons on this kind of field effect transistors.

In this paper, we study the transistor performance of germanene nanoribbons with different widths in the channel and leads by the combination of density functional theory (DFT) and Non-equilibrium Green's function (NEGF) method. The output and transfer characteristics of the proposed FETs are investigated and then analyzed for both analog and digital applications.

\section{Device structure and simulation procedure}

The open-source ab initio package named TransSIESTA [25] based on density functional method and non-equilibrium Green's function (NEGF) formalism is used to obtain the electronic structure and current-voltage characteristics of germanene nanoribbons (GeNRs) with different widths. Double- $\zeta$ plus polarization (DZP) sets are employed as the linear combination of atomic orbitals (LCAO) basis sets with Perdew, Burke, and Ernzerhof (PBE) [26, 27] exchange-correlation 
functional. Orbitals of Kohn-Sham are expanded up to 150Ry and a vacuum thickness of $15 \AA$ and $25 \AA$ are applied in the $\mathrm{x}$ and $\mathrm{y}$-directions to eliminate the artificial nanoribbons. All atoms in the unit cell of the ribbon are relaxed until a force convergence of $0.02 \mathrm{eV} / \AA$ is achieved. A sampling of $1 \mathrm{x} 1 \mathrm{x} 11$ and $1 \times 1 \times 101 \mathrm{k}-$ mesh are used to optimize the structures and obtain the accurate ground state structures, respectively.

Fig. 1(a) shows the unit cell of an armchair GeNR with $n=14$ and $\Delta=0.65 \AA$ buckling parameter which is used as leads of the proposed field-effect transistors (FETs) which shows schematically in Fig. 1(b). In order to utilize bandgap engineering of the channel, germanene nanoribbons with $n=12$ and 16 are used. Hydrogen atoms are attached to the ribbon edges of the GeNRs to saturate the dangling bonds.

(b)

(a)
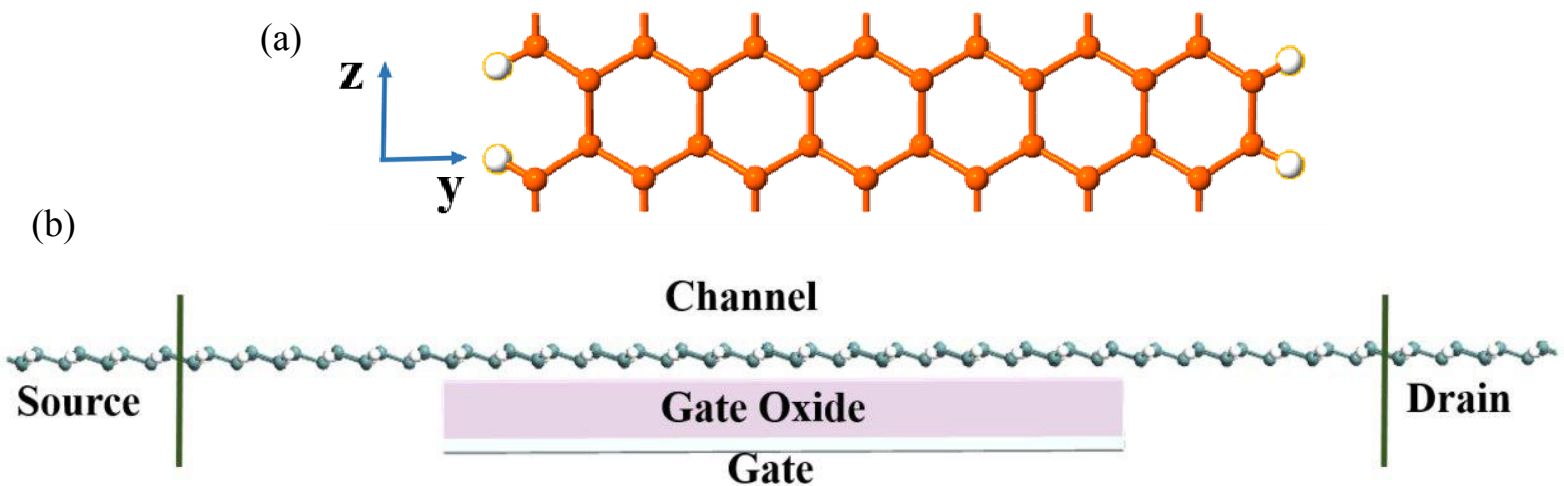

Fig 1. (a) The unit cell of germanene nanoribbon with hydrogen passivation. (b) Schematic sketch of the considered field effect transistor.

Investigation of electron transport through the device is performed by selfconsistent calculation of DFT and NEGF.

(a)

(b)
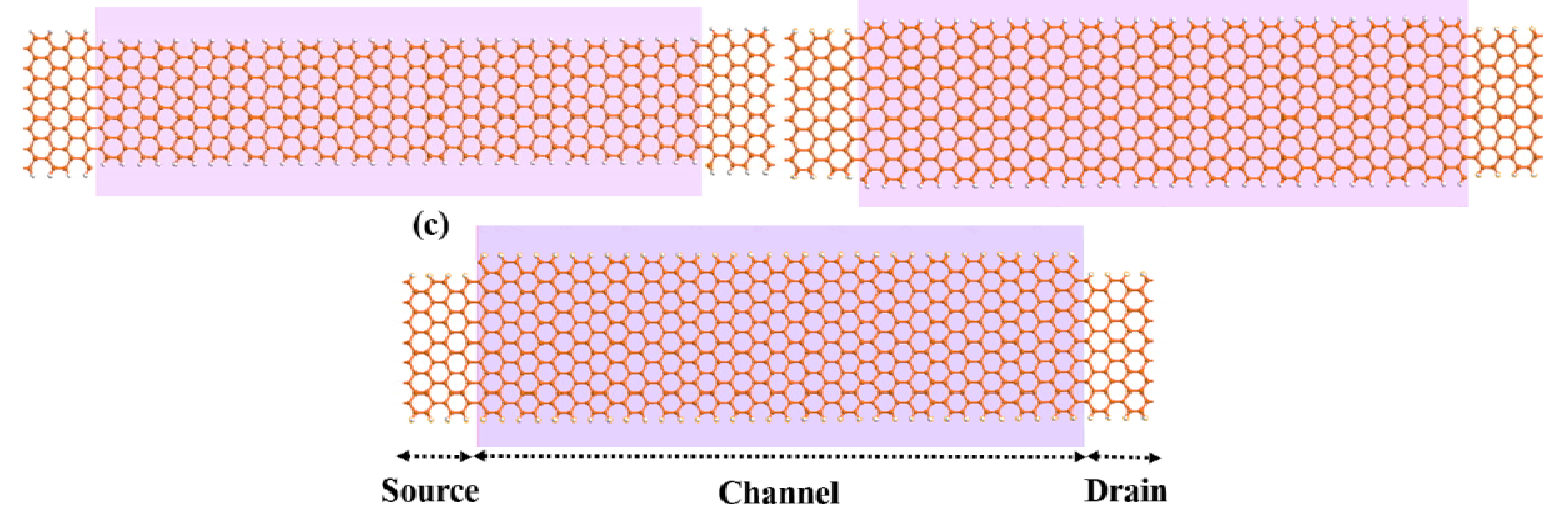

Fig 2. Configurations of channel; thin (a), thick (b) and T (c). The pink square behind the structures is metal gate. 
We consider a $10 \mathrm{~nm}$ GeNR as the channel of the proposed devices. Fig. 2 shows the gated proposed GeNR-FETs with different channel widths. We consider three different configurations for the channel of the FETs which are named thin $(n=12)$, thick $(n=16)$ and T-shape $(n=16)$ structures. Landauer-Buttiker formula [28] with self-consistent approach is employed to calculate electron transport through the channel of the devices, (Eq. 1):

$I\left(V_{g}, V_{\text {bias }}\right)=\frac{2 e}{h} \int_{-\infty}^{+\infty}\left\{T\left(E, V_{g}, V_{\text {bias }}\right)\left[f_{L}\left(E-\mu_{L}\right)-f_{R}\left(E-\mu_{R}\right)\right]\right\} d E(1)$

Where $\mathrm{T}\left(\mathrm{E}, \mathrm{V}_{\mathrm{g}}, \mathrm{V}_{\text {bias }}\right), \mathrm{Vg}, \mathrm{V}_{\text {bias }}, \mathrm{f}_{\mathrm{L} / \mathrm{R}}$ and $\mu_{\mathrm{L}} / \mu_{\mathrm{R}}$ are the transmission probability, gate voltage, Drain-Sourcebias voltage, Fermi-Dirac distribution function for the left $(\mathrm{L}) /$ right $(\mathrm{R})$ electrode and the electrochemical potential of the $\mathrm{L} / \mathrm{R}$ electrodes, respectively.

\section{Results and discussion}

In this section, we analyze the obtained results from two point of views: analog and digital applications. Hence, we present the transfer characteristics and output characteristics of the simulated devices at room temperature and we investigate three different figures of merit including $\mathrm{I}_{\text {on }} / \mathrm{I}_{\text {off }}$ ratio,negative differential conductance and peak-to-valley (PVR) ratio.

\subsection{Transfer characteristics}

In this subsection, we investigate and compare the transfer characteristics of three simulated devices atthe ballistic regime. In all devices, we consider a germanene nanoribbon as source and drain leads where both have the same width with $\mathrm{N}=14$ germanium atoms. While the channel region includes a germanene nanoribbon with $\mathrm{N}=12$ and 16 germanium atoms as depicted in Fig. 2. In the case of the channel with $\mathrm{N}=16$ atoms, we consider two configurations; T-shape and wide (symmetric) channels.

Fig. 3 illustrates the current through the channel as a functionof the gate voltagefor all three simulated Ge-NRFETs with thin, wide and T-shape channels. The figure shows that each current curve displays a minimum value at almost the same value of the gate voltage i.e. $\mathrm{V}_{\mathrm{g}} \sim 0.1$ V.Moreover, it reflects two points about the ONand OFF- current for all three devices. As can be seen, the maximum value of the currenthas not changed significantly, while the minimum current decreases by 
changing the width and shape of the channel region from T-shape to thick and then thin cases.

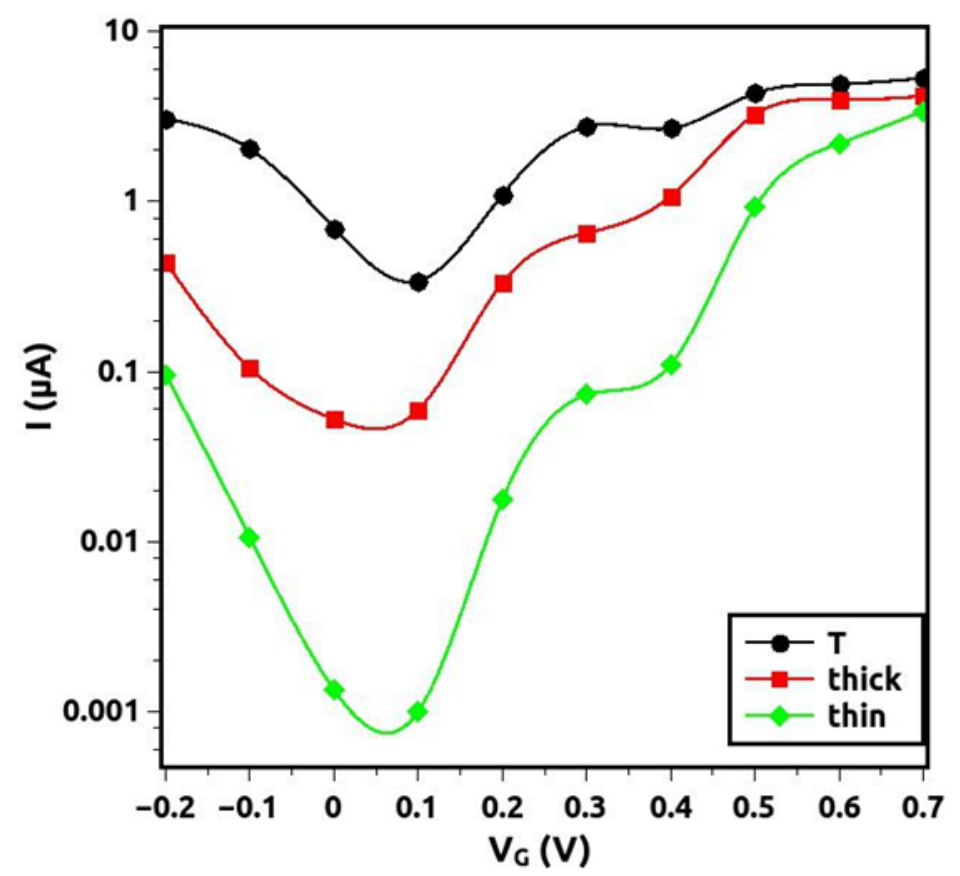

Fig 3. The current as a function of the gate voltage for all three simulation Ge-NRFETs.

As ref. [15] demonstrates, the relationship between the band gap and width of an armchair Ge-nanoribbon can be classified in three categories: $N=3 \mathrm{~m}, \mathrm{~N}=3 \mathrm{~m}+1$ and $\mathrm{N}=3 \mathrm{~m}+2$; where $\mathrm{N}$ denotes the number of germanium atoms and $\mathrm{m}$ is an integer. Thus, $\mathrm{N}=3 \mathrm{~m}+2$ has the least band gap. Fig. 4, shows the band gap of three germanene nanoribbons with different widths of $\mathrm{N}=12,14,16$. In our simulations and in all three devices, the source and drain leads are selected as armchair Genanoribbon with the same width $(\mathrm{N}=14)$ which follows the $\mathrm{N}=3 \mathrm{~m}+2$ category. For the channel, we consideredthree cases: $\mathrm{N}=12$ (thin channel) which falls inthe $\mathrm{N}=3 \mathrm{~m}$ category and $\mathrm{N}=16$ which corresponds to the $\mathrm{N}=3 \mathrm{~m}+1$ category. The channel with $\mathrm{N}=16$ is considered in two situations; the $\mathrm{T}$-shape and the wide (symmetric) shape. 


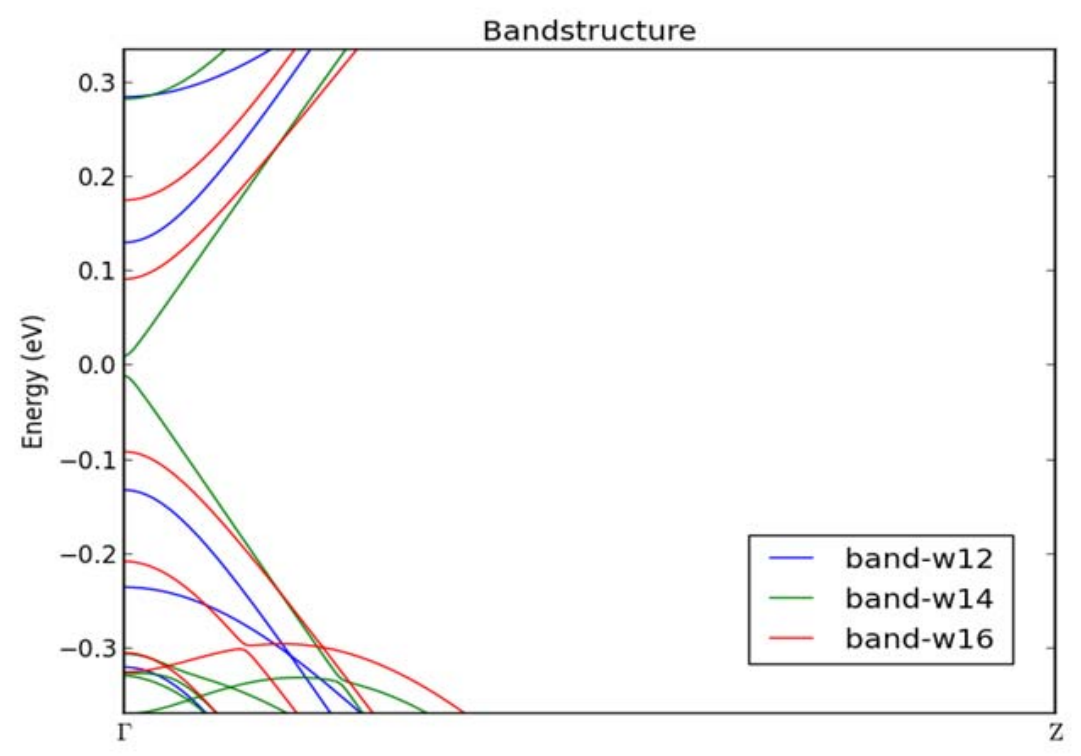

Fig 4.The band gap of three germanene nano ribbon with different width $\mathrm{N}=12,14,16$.

With this engineering on the width of the armchair Ge-nanoribbon in the source, drain and channel regions of the GeNR-FET, we see that the ON- current in transfer characteristics are almost the same for three devices. This issue is justified by the fact that the ON- current more depends onthe band gap in the source and drain regions and less affected by the channel states. So, it is suitable that the width of these regions selected from the $\mathrm{N}=3 \mathrm{~m}+2$ category with negligible band gap. On the other hand, by selecting the germanene nanoribbon with wider band gap for the channel region, we see that a dip appears near the Dirac point in all three devices which display that the band gap in the channel region has a key role in creating the dip in transfer characteristics. As the thin channel case $(\mathrm{N}=12)$ curve shows, the larger band gap in the channel region leads to lower OFF- current and improved $\mathrm{I}_{\text {on }} / \mathrm{I}_{\text {off }}$ ratio.

Also, from transfer characteristics, we see that in the T-shape channel and the wide channel cases the width and the band gap of the ribbon in the channel region are same, but the minimum current in T-shape channel case is higher than the wide channel case. This feature originates from the difference in the position of the germanium atoms in the edge of the interface between the channel and source/drain regions in two structures. In Fig. 5, we see that in the T-shape channel case as presented in the figure, some edge of the germanium atoms have two bonds 
with neighbor atoms and therefore each of these atoms have two free electrons while in the wide channel case, fewer atoms have this situation. This results that the T-shape channel case has more free electrons in the channel region and this electrons in turn increase the conductivity and thus decrease the minimum current in T-shape channel.
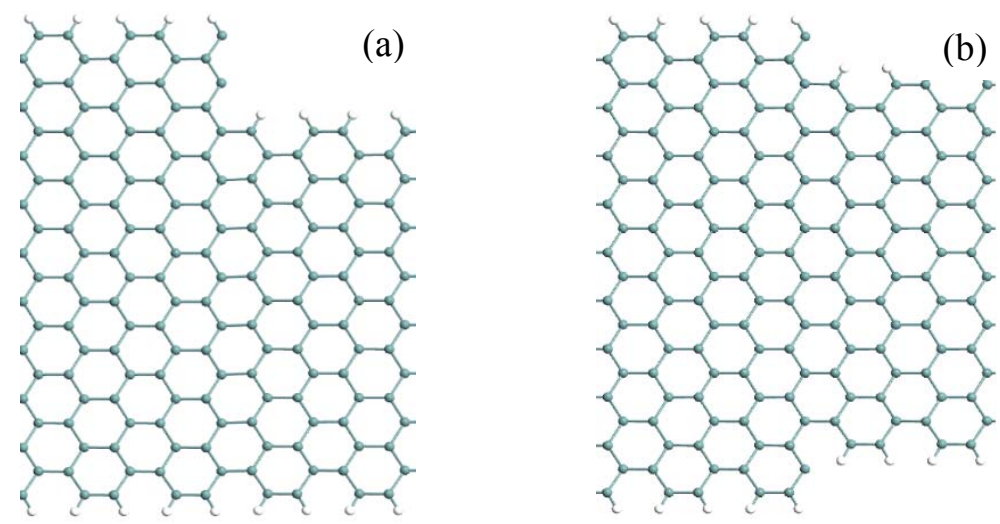

Fig 5. (a) The T-shape and (b) the wide channel interface with drain region.

To better understand of this issue in Fig .6, we plot the pathway of the electrons in (a) T- shape channel case and (b) the wide channel case GeNR-FET.

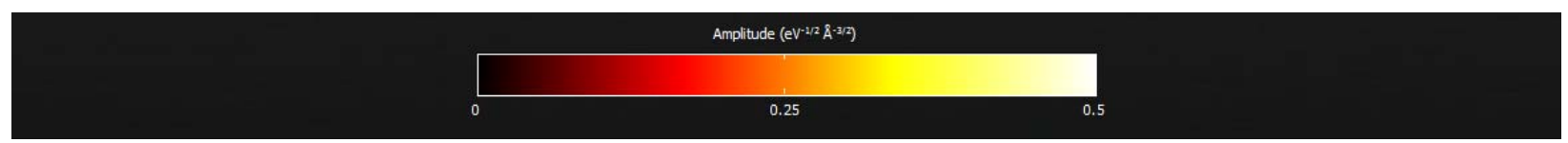

(a)

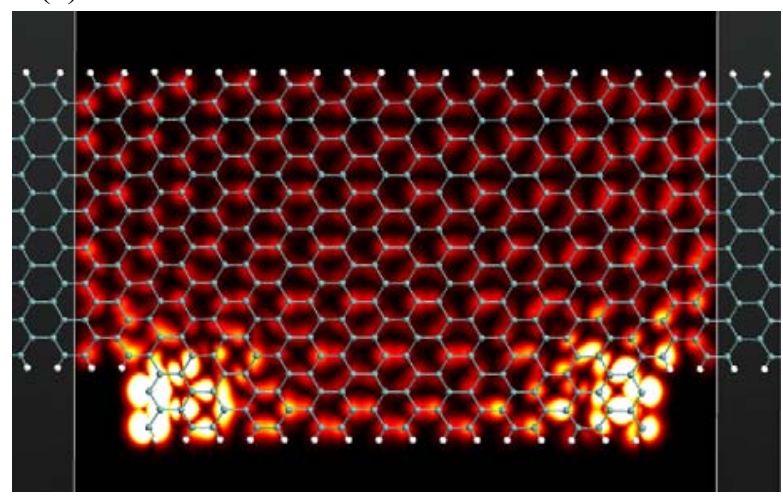

(b)

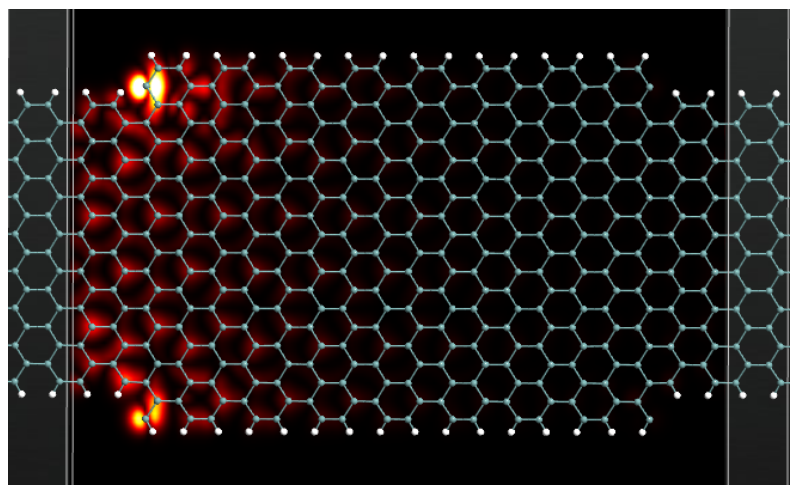

Fig 6. The pathway of the electrons in (a) T- shape channel case and (b) the wide channel case GeNR-FET.

In this plot, the brightness shows the electron density in this two structures. We see clearly that the density of carriers and the conductivity in corners of the channel in the T-shape channel case is much more than the wide channel case. 
From results and due to the higher $\mathrm{I}_{\text {on }} / \mathrm{I}_{\text {off }}$ ratio, it is concluded that the case of thin channel geometry is more appropriate for digital applications.

\subsection{Output characteristics}

Now we investigate the output characteristics of the simulated devices. This time due to the study of the analog application in all three devices, we consider anarmchairgermanene nanoribbon with width of $\mathrm{N}=16$ atoms which corresponds to $\mathrm{N}=3 \mathrm{~m}+1$ category forthe source and drain leads. In the T-shape channel and wide channel, we consider anarmchairgermanene nanoribbon with the width of $\mathrm{N}=20$ from the $\mathrm{N}=3 \mathrm{~m}+2$ category with small band gap and in the thin channel, the number of germanium atoms in the width of the ribbon is chosen as $\mathrm{N}=12$ which fall in $\mathrm{N}=3 \mathrm{~m}$ category. In Fig. 7, we plot the current as a function of the bias voltage for all three simulated devices. The gate voltage is chosen $\mathrm{V}_{\mathrm{g}}=0.3 \mathrm{~V}$ andthe results are obtained instandard room temperature.

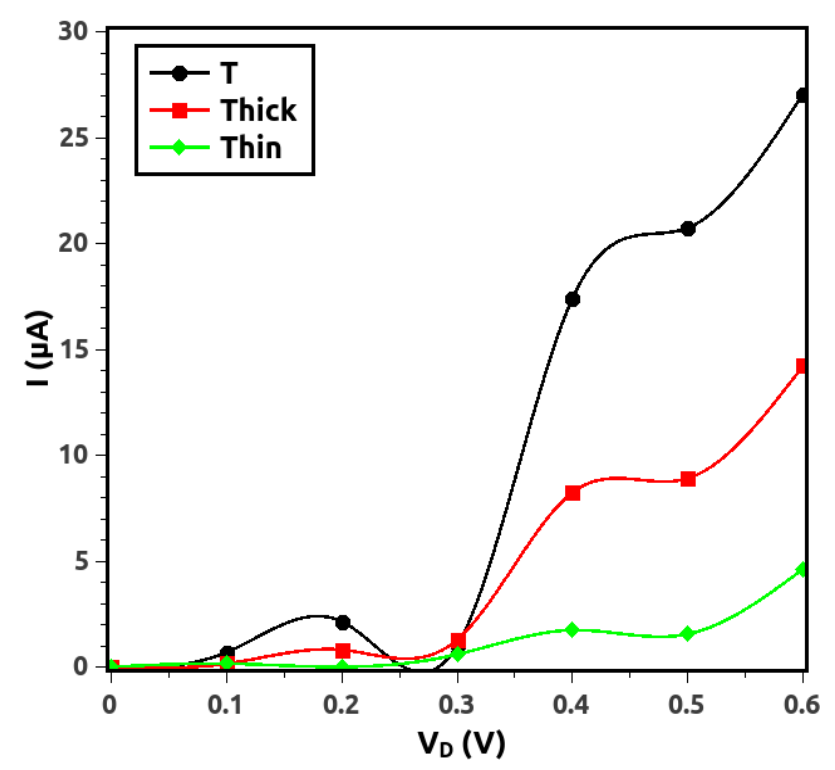

Fig 7. The current density as a function of the bias voltage for all three simulated devices.

As shown in Fig.7, for all three devices, firstly by increasing the bias voltage there is no current through the devices. By further increase of the bias voltage the current shows a small peak and immediately the current decreases and then increases rapidly which show clearly a negative differential conductance (NDC) at the almost same bias value for all three devices. 
The origin of the output characteristics behavior can be understood as follow.At zero bias voltage, the Dirac point of thebandstructure of the source and drain regions are aligned while due to the topgate voltage, the Dirac point of the channel region is situated higher. However, due tothe buckled structure of armchair germanene nanoribbon,there is an intrinsicband gap around the Dirac point. Therefore, by increasing the drain voltage from zero to $0.07 \mathrm{~V}$,the Fermi level lies in band gap region and there is no current flows for all three cases. By further increase of the bias voltage, after overtaking the band gap region an energy window will openand the channel and drain bands move downward. Therebythe Fermi energy lies in aregion near the top of the valence band with the lower density ofstates and thus a small peak appears and then with fall of the Fermi level in the channel band gap, the current instantly decreases. Further increase of the bias voltage causes the Fermi level to lie in the conduction band of the channel regionand thus, the current will rapidly be increased and a great peak appears. But, as can be seen from Fig.7, the peak current of the T-shape channel is larger than two other cases.

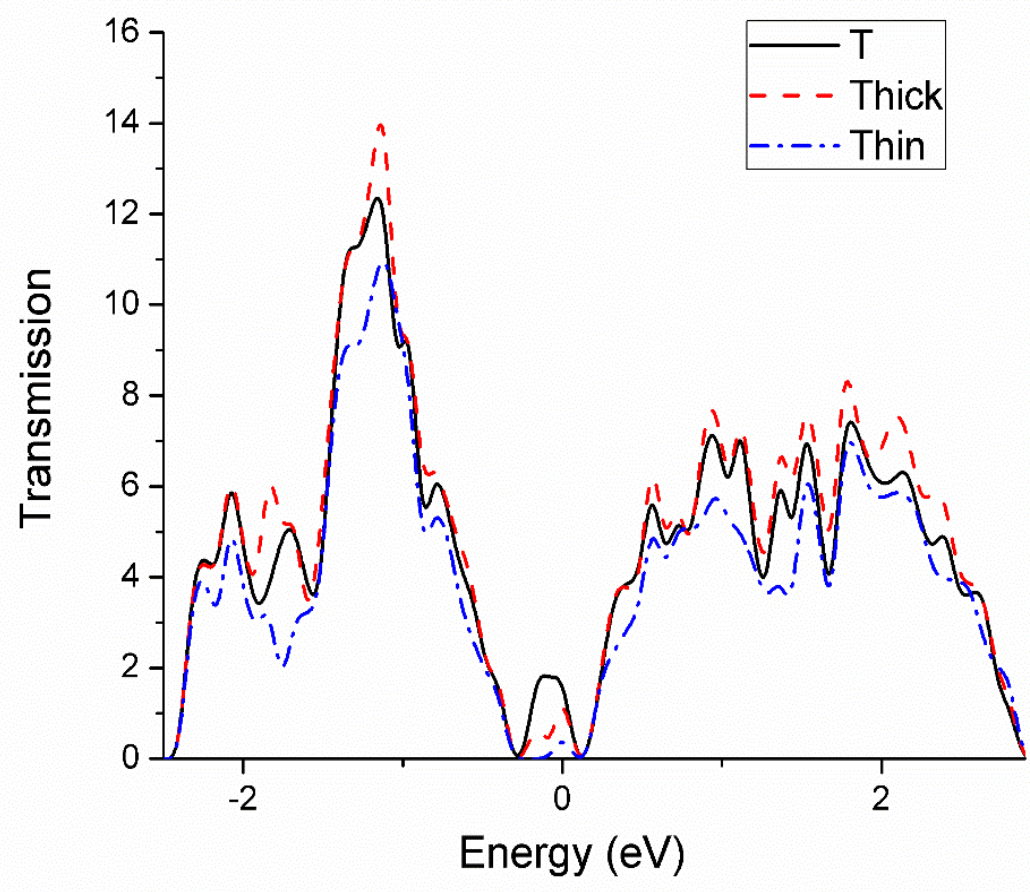

Fig8. The transmission spectrum for all three device at $\mathrm{V}_{\mathrm{ds}}=0.4 \mathrm{~V}$ and $\mathrm{V}_{\mathrm{g}}=0.3 \mathrm{~V}$. 
To understand the reasons for this behavior we analyze the transmission spectrum of all three devices at the bias voltage which the peak appeared in output characteristics diagram. Fig. 8, shows the transmission spectrum for all three devices at $\mathrm{V}_{\mathrm{ds}}=0.4 \mathrm{~V}$ and $\mathrm{V}_{\mathrm{g}}=0.3 \mathrm{~V}$. As can be seen from the figure, the transmission value which corresponds to chiral tunneling around the Fermi level $(E=0)$, shows higher value for $T$-shape channel case compared with two other cases. It comes from the transition states in the channel region for the T-shape channel which is wider than the other ones and thus leads to greater transmission in chiral tunneling regime. By comparison between the wide and the thin channels, results show that by selecting the nanoribbon with finite bandgap in the source and drain regions andsmallbandgap in the channel region, we should be ableto strongly enhance the peak to valley ratio (PVR) from 2.94 in the thin channel case to 6.43 in the wide channel case. However, when comparing the output characteristic of the T-shape channel and the wide channel cases, it is clear that by engineering of the shape of the channel region, the transmission spectra improves. It can be possible to reach a larger peak to valley (PVR) ratio up to 17.28 in T-shape channel case. Higher PVR is desirable for analog applications.

\section{Conclusion}

In summary, in this paper, we simulated a field effect transistor based on germanene nanoribbon and using the advantage of engineering the nanoribbon width in the source, drain and channel regions. We investigate the effect of the width and geometry of the ribbon on transistor performance. The transfer characteristic results showed that the GeNR-FET from the $N=3 m$ and $N=3 m+1$ categories with a finite band gap in the channel region and from the $\mathrm{N}=3 \mathrm{~m}+2$ category in the source/drain with a small band gap shows better $\mathrm{I}_{\mathrm{on}} / \mathrm{I}_{\mathrm{off}}$ ratio rather than two other cases.However, the output characteristic revealed that the GeNRFET with the finite band gap in the source/drain regions and small band gap in the channel region shows better operation in PVR. Also, we find the PVR for the Tshape channel case reaches to 17.28. Our research showed that a better operationcan be achieved in eitheranalog or digital applications by tuning the width and geometry of the germanenenanoribbon in each regions of the GeNRFET.

\section{References:}

[1] K. S. Novoselov, A. K. Geim, S. V. Morozov, D. Jiang, Y. Zhang, S. V. Dubonos, I. V. Grigorieva, and A. A. Firsov, Science, 2004, 306: 666 
[2] K. S. Novoselov, A. K. Geim, S. V. Morozov, D. Jiang, M.I. Katsnelson, I. V. Grigorieva, S. V. Dubonos, and A. A. Firsov, Nature (London), 2005, 438: 197

[3] C. Berger, Z. M. Song, X. B. Li, X. S. Wu, N. Brown, C. Naud, D. Mayou, T. B. Li, J. Hass, A. N. Marchenkov, A.H. Conrad, P. N. First, and W. A. de Heer, Science, 2006, 312: 11915. T. Ohta, A. Bostwick, T. Seyller, K. Horn, and E. Rotenberg, Science, 2006, 313: 951

[4] K. S. Novoselov, A. K. Geim, S. V. Morozov, D. Jiang, Y. Zhang, S. V. Dubonos, I. V. Grigorieva and A. A. Firsov, Science, 2004, 306, 666-669.

[5] Miro, Pere, Martha Audiffred, and Thomas Heine. "An atlas of twodimensional materials." Chemical Society Reviews 43.18 (2014): 6537-6554.

[6] Schwierz, F., J. Pezoldt, and R. Granzner. "Two-dimensional materials and their prospects in transistor electronics." Nanoscale 7.18 (2015): 8261-8283.

[7] K. Takeda and K. Shiraishi, Phys. Rev. B 50, 14916 (1994).

[8] S. Cahangirov, M. Topsakal, E. Aktürk, H. Şahin, and S. Ciraci, Phys. Rev. Lett. 102, 236804 (2009).

[9] P. Vogt, P. De Padova, C. Quaresima, J. Avila, E. Frantzeskakis, M. C. Asensio, A. Resta, B. Ealet, and G. Le Lay, Phys. Rev. Lett. 108, 155501(2012). [10] J.-A. Yan, R. Stein, D. M. Schaefer, X.-Q. Wang, and M. Y. Chou, Phys. Rev. B 88, 121403(R) (2013).

[11] Takeda K and Shiraishi K 1994 Theoretical possibility of stage corrugation in $\mathrm{Si}$ and Ge analogs of graphite Phys. Rev. B 5014916

[12] Ritter K A and Lyding J W 2009 The influence of edge structure on the electronic properties of graphene quantum dots and nanoribbons Nat. Mater. 8 235-242

[13] Mativetsky J M, Liscio A, Treossi E, Orgiu E, Zanelli A, Samori P and Palermo V J 2011 Graphene transistors via in situ voltage-induced reduction of graphene-oxide under ambient conditions Am. Chem. Soc. 133 14320-14326

[14] Han M Y, Ozyilmaz B, Zhang Y B and Kim P 2007 Energy band gap engineering of graphene nanoribbons Phys. Rev. Lett. 98 206-805

[15] Shiro Kaneko, Hideaki Tsuchiya, Yoshinari Kamakura, Nobuya Mori, Matsuto Ogawa Theoretical performance estimation of silicene, germanene, and graphene nanoribbon field-effect transistors under ballistic transport Applied Physics Express 7, 035102 (2014).

[16] A.H. Bayani, D. Dideban, M. Vali, N. Moezi, Germanene nanoribbon tunneling field effect transistor (GeNR-TFET) with a $10 \mathrm{~nm}$ channel length: analog performance, doping and temperature effects, Semicond. Sci. Technol. 31 (2016) 4. 
[17] A.H. Bayani, D. Dideban, N. Moezi, Hydrogen sensitive field-effect transistor based on germanene nanoribbon and optical properties of hydrogenated germanene, J. Comput. Electron 15 (2) (2016) $381 \mathrm{e} 388$.

[18] Bolotin K. I., Sikes K. J., Jiang J., Klima M., Fudenberg G., Hone J., Kim P. and Stormer H. L. 2008 Solid State Commun. 146351

[19] Zomer P. J., Dash S. P., Tombros N. and van Wees B. J. 2011 Appl. Phys. Lett. 99232104

[20] Novoselov K. S., Geim A. K., Morozov S. V., Jiang D., Zhang Y., Dubonos S. V., Grigorieva I. V. and Firsov A. A. 2004 Science 306666

[21] A.H. Bayani, D. Dideban, N. Moezi, Impact of uniaxial compressive strain on physical and electronic parameters of a $10 \mathrm{~nm}$ germanene nanoribbon field effect transistor, Superlattices and Microstructures, 100 (2016) 198-208.

[22] H. Mizuta, T. Tanoue, "The Physics and Application of Resonant Tunneling Diodes" (Cambridge: Cambridge University Press 1995.

[23] Viet Hung Nguyen, Alfonso Alarcon, Salim Berrada, Van Nam Do, Jerome Saint-Martin, Philippe Dollfus, Damien Querlioz, Arnaud Bournel On the nonlinear effects in graphene devices J. Phys. D: Appl. Phys. 47 (2014) 094007.

[24] Viet Hung Nguyen, Jérôme Saint-Martin, Damien Querlioz, Fulvio Mazzamuto, Arnaud Bournel, Yann-Michel Niquet, Philippe Dollfus, "Bandgap nano engineering of graphene tunnel diodes and tunnel" transistors to control the negative differential resistance J Comput Electron (2013) 12:85-93.

[25] Soler, J.M., Artacho, E., Gale, J.D., Garcia, A., Junquera, J., Ordejon, P., Portal, D. S.: The SIESTA method for ab initio order $\mathrm{N}$ materials simulation. J. Phys. Condens. Matter 2002, 14, 2745-2779.

[26] Perdew, J.P., Chevary, J.A., Vosko, S.H., Jackson, K.A., Pederson, M.R., Singh, D.J., Fiolhais, C. Atoms, molecules, solids, and surfaces: applications of the generalized gradient approximation for exchange and correlation. Phys. Rev. B. 1992, 46, 6671-6687.

[27] Perdew, J.P., Burke, K., Wang, Y. Generalized gradient approximation for the exchange-correlation hole of a many electron system. Phys. Rev. B. 1996, 54, $16533-16539$.

[28] Brandbyge, M.; Mozos, J. L.; Ordejon, P.; Taylor, J.; Stokbro K.; Density functional method for nonequilibrium electron transport. Phys. Rev. B 2002, 65, 165401. 